IAC -07- A6.3.01

\title{
THE CHARACTERISTICS AND CONSEQUENCES OF THE BREAK-UP OF THE FENGYUN-1C SPACECRAFT
}

\author{
Nicholas L. Johnson \\ NASA Johnson Space Center, Houston, TX, USA \\ nicholas.l.johnson@nasa.gov \\ E. Stansbery \\ NASA Johnson Space Center, Houston, TX, USA \\ eugene.g.stansbery@nasa.gov \\ J.-C. Liou \\ ESCG/ERC, Houston, TX USA \\ jer-chyi.liou1@jsc.nasa.gov \\ M. Horstman \\ ESCG/ERC, Houston, TX USA \\ matt.horstman-1@nasa.gov \\ C. Stokely \\ ESCG/Barrios Technology, Houston, TX USA \\ christopher.l.stokely@nasa.gov \\ D. Whitlock \\ ESCG/Hamilton Sundstrand, Houston, TX USA \\ david.o.whitlock@nasa.gov
}

\begin{abstract}
The intentional break-up of the Fengyun-1C spacecraft on 11 January 2007 via hypervelocity collision with a ballistic object created the most severe artificial debris cloud in Earth orbit since the beginning of space exploration. More than 2000 debris on the order of $10 \mathrm{~cm}$ or greater in size have been identified by the U.S. Space Surveillance Network. The majority of these debris reside in long-lived orbits. The NASA Orbital Debris Program Office has conducted a thorough examination of the nature of the Fengyun-1C debris cloud, using SSN data for larger debris and special Haystack radar observations for smaller debris. These data have been compared with the NASA standard satellite break-up model for collisions, and the results are presented in this paper. The orbital longevity of the debris have also been evaluated for both small and large debris. The consequent long-term spatial density effects on the low Earth orbit regime and the potential effect of the debris cloud on the growth of the near-Earth satellite population have been addressed.
\end{abstract}

\section{BACKGROUND}

The Fengyun-1C meteorological spacecraft (International Designator 1999-025A, U.S. Satellite Number 25730) was launched on 10 May 1999 into a nearly circular, sunsynchronous orbit with a mean altitude of approximately $850 \mathrm{~km}$ and an inclination of 98.8 degrees. This altitude regime has long been used for monitoring Earth's global weather patterns. Current operational spacecraft of the U.S. NOAA (National Oceanic and Atmospheric Administration) and DMSP (Defense Meteorological Satellite Program) networks 
employ orbits virtually identical to that of Fengyun-1C. Russian meteorological spacecraft of the Meteor 1 and Meteor 2 series also used this altitude regime in the 1970's and 1980's in supplementary inclinations of 81.2 degrees.
The basic configuration of the nearly 960-kg spacecraft was a cube with a side dimension of approximately $1.5 \mathrm{~m}$ and a pair of solar arrays, each about $1.5 \mathrm{~m}$ wide and $4 \mathrm{~m}$ long (Figure 1).

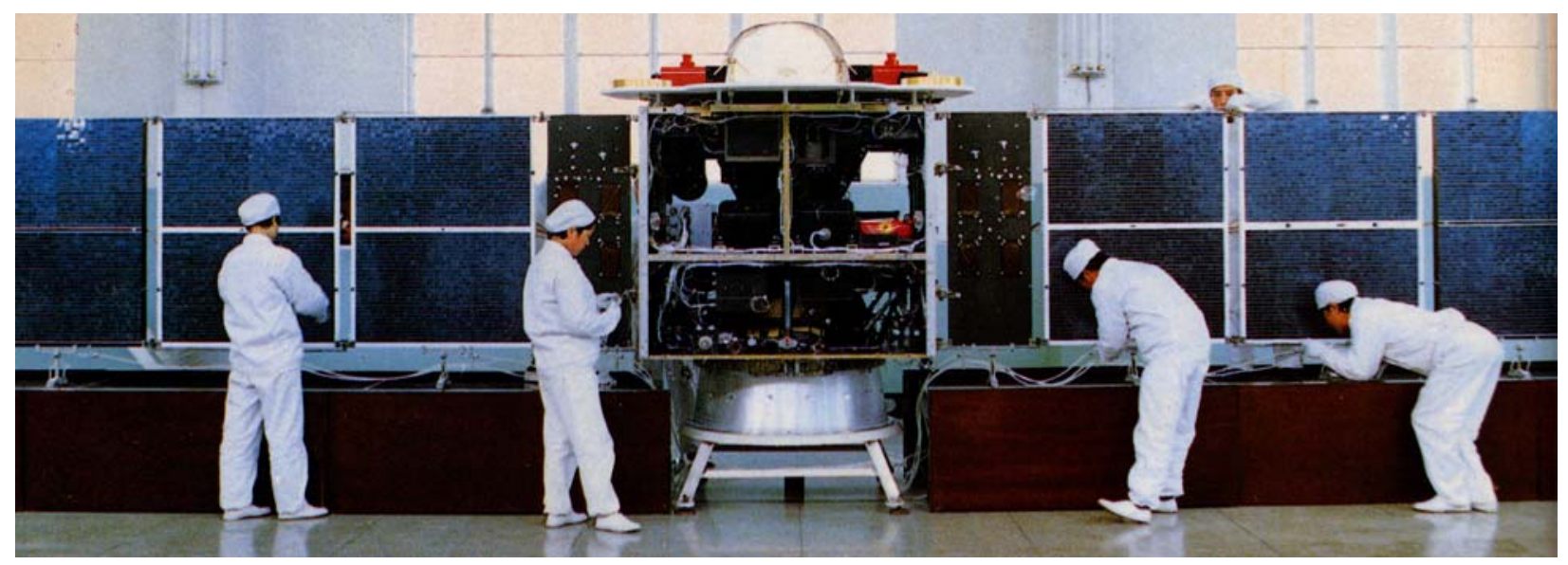

Fig. 1. A Fengyun-1 satellite in final assembly. The vehicle is shown in an inverted position; the sensors at the "top" point toward the Earth.

A replacement for Fengyun-1C, the Fengyun-1D spacecraft, was launched in May, 2002, but Fengyun-1C considerably exceeded its 2-year design life by several years, working through at least 2005. By January 2007 the spacecraft was reportedly still responding to Chinese controllers but was no longer providing significant meteorological services.

As Fengyun-1C was southbound over central China on 11 January 2007, the spacecraft was intercepted by a ballistic kinetic kill vehicle (KKV) reportedly launched from or near to the Xichang space launch center. The event was first publicly disclosed on 17 January by the U.S. periodical Aviation Week and Space Technology and later acknowledged by Chinese government officials on 23 January. ${ }^{1-2}$ Subsequent official Chinese statements referred to the test but provided no technical information.

According to published reports, the disintegration of Fengyun-1C occurred at 2226 GMT on 11 January (12 January in China); this time is consistent with an analysis of the debris cloud and the orbit of Fengyun-1C prior to the break-up. ${ }^{3}$ At that time the spacecraft was about $860 \mathrm{~km}$ above China near $35^{\circ} \mathrm{N}, 100^{\circ} \mathrm{E}$, northwest of the vicinity of Xichang $\left(28^{\circ} \mathrm{N}\right.$, $\left.102^{\circ} \mathrm{E}\right)$. With an assumption of a ballistic trajectory from Xichang with an apogee near 860 $\mathrm{km}$, the relative collision velocity of the KKV with Fengyun-1C would have been approximately $9 \mathrm{~km} / \mathrm{s}$, i.e.., a hypervelocity impact.

\section{DEBRIS CLOUD CHARACTERIZATION}

To understand the extent and the characteristics of the Fengyun-1C orbital debris cloud, data from a variety of sources were assembled and analyzed. By far the most important of these data sources was the U.S. Space Surveillance Network (SSN). Information from the SSN not only provided explicit piece counts for debris larger than $5 \mathrm{~cm}$ but also permitted the assessment of debris size and area-to-mass ratios. Data on even smaller debris were collected by the Haystack and Goldstone radars, operated by the Massachusetts Institute of Technology's (MIT) Lincoln Laboratory and the California Institute of Technology's Jet Propulsion Laboratory, respectively.

By integrating the information from these various sensor systems, the potential effect of the Fengyun-1C debris cloud on near-term and far- 
term operational space systems and the orbital debris environment in general can be assessed.

\section{Data from the U.S. Space Surveillance Network}

The first data on orbital debris from the break-up of Fengyun-1C, in the form of 32 two-line element sets (TLEs), were released by the SSN on 18 January. However, nearly 600 additional debris had already been positively identified by that date. With each tracking of the debris cloud by the SSN radars, a careful correlation process was necessary to assign individual observations to the proper debris piece.

Due to the additional manual task of formally cataloging the debris, the official number of debris identified with the cloud was always less than the total number of debris being tracked. By 11 July (six months after the test), the number of cataloged debris had reached 1967, while the total number of debris being tracked exceeded 2300 (Figure 2).

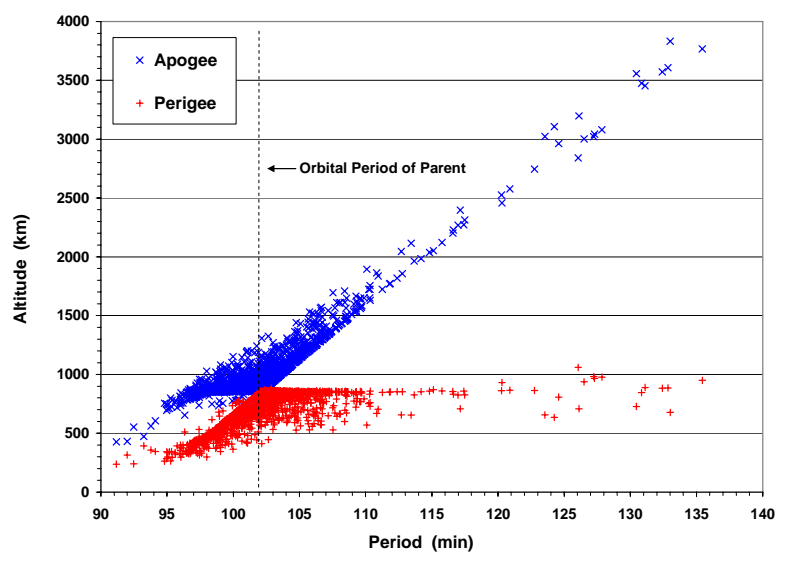

Fig. 2. A Gabbard diagram (apogee and perigee versus orbital period) of the Fengyun-1C orbital debris cloud as assessed on 11 July 2007, six months after the break-up with a total of 2347 objects individually identified.

The debris appear to have been ejected asymmetrically with respect to the original velocity vector of the spacecraft. While the larger number of debris with orbital periods greater than that of Fengyun-1C prior to the break-up is due primarily to the immediate reentry of debris with high retrograde ejection velocities, a slight posigrade asymmetry ( $60 \%$ to $40 \%$ ) does exist for debris within the heart of the cloud. The asymmetry in inclination (velocity component perpendicular to the plane of the Fengyun-1C orbit) is much more pronounced, i.e., $\quad 20 \%$ toward higher inclinations (Figure 3).

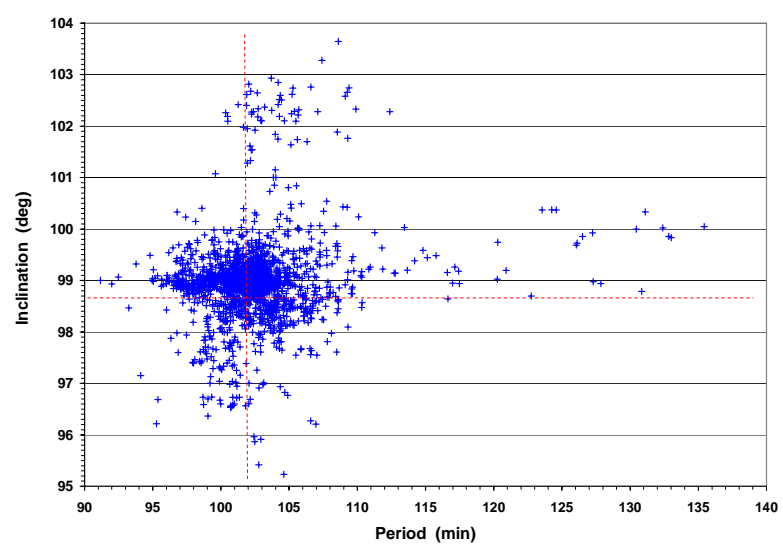

Fig. 3. Approximately $80 \%$ of the tracked debris were found in inclinations greater than those of the spacecraft prior to impact.

The size of each fragment is determined by converting radar cross-sectional (RCS) data from SSN sensors to a characteristic length with the NASA Size Estimation Model (SEM). ${ }^{4}$ The smallest size a radar can reliability detect is a function of both its frequency and its power. Due to attitude instabilities of the debris and individual radar peculiarities, RCS values can vary between radars and even between different passes of a single radar. However, size estimations involving a large ensemble of debris can prove very helpful in analyzing a satellite break-up.

A preliminary analysis of RCS data from the SSN suggests that over 1600 of the Fengyun-1C debris appear to possess a characteristic length in excess of $10 \mathrm{~cm}$. By comparison, the current NASA standard break-up model would predict about 1000 debris of that size remaining in orbit after the first day. Whereas individual events can easily differ from the "average" fragmentation of the NASA standard break-up model, the initial debris size estimates of the SSN have yet to be confirmed. Still to be resolved are some potential biases between radars for small debris, i.e., smaller than $20 \mathrm{~cm}$. It is possible that some 
of the early debris size estimates are larger than the actual size (see next section for additional insight on this topic). Hence, a final comparison of the Fengyun-1C debris and the NASA standard break-up model remains to be completed.

An important feature of a break-up fragment, which affects its potential long-term effect on the near-Earth environment, is its area-to-mass ratio $(\mathrm{A} / \mathrm{M})$. The larger the $\mathrm{A} / \mathrm{M}$ the more rapidly it normally falls back to Earth due to atmospheric drag and solar radiation pressure effects. The $\mathrm{A} / \mathrm{M}$ of an object can be estimated by monitoring its orbital behavior over time. Numerous Fengyun-1C debris exhibit large $\mathrm{A} / \mathrm{M}$ values, which will result in shorter orbital lifetimes than those for typical satellite break-up debris. Figure 4 indicates the distribution of $\mathrm{A} / \mathrm{M}$ values from the Fengyun-1C debris as a function of apparent characteristic length.

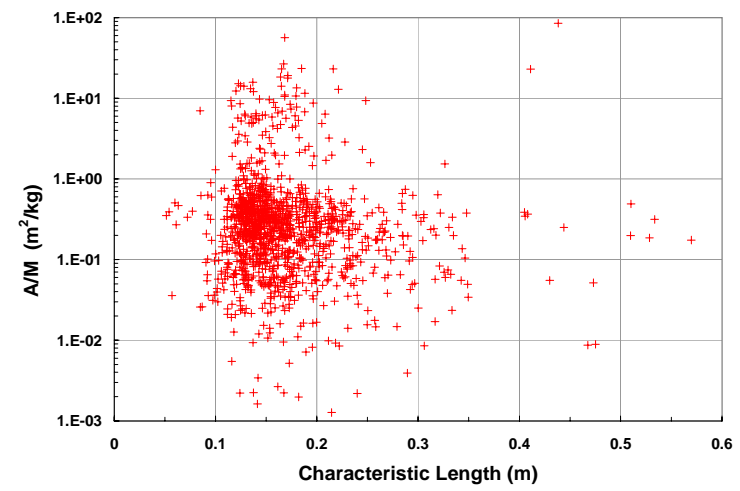

Fig. 4. Estimated area-to-mass $(\mathrm{A} / \mathrm{M})$ ratios as a function of debris characteristic length.

\section{Data from the Haystack Radar}

The Fengyun -1C debris cloud was also observed by the Haystack radar. Haystack has been NASA's primary source of data for centimetersize debris since 1990. It is an X-band dish radar operated at 3-cm wavelength with a very high sensitivity (18 dB signal-to-noise on a 1-cm sphere at $1000-\mathrm{km}$ slant range), but a narrow $\left(0.058^{\circ}\right)$ field-of-view. Approximately 24 hours after the break-up, Haystack observed the debris cloud using an "orbit plane tracking” operational mode where Haystack was directed to observe the orbit plane of Fengyun-1C parent spacecraft using orbital parameters propagated from the last TLE available prior to the break-up. The orbit plane was observed for $\sim 1.9$ hours starting just after 2000 GMT on January 12, 2007 as it passed over the radar site from east to west.

During this observation period, the elevation angle of the radar varied between $22^{\circ}$ and $50^{\circ}$. Because of this changing geometry, the range from the radar to the orbit plane, and hence the limiting debris size which the radar could detect, varied with time. However, the radar reliably detected 1-cm and larger objects over the entire range of the measurement and detected objects half that size at closer ranges, when the elevation angle was at its highest and the orbit plane was passing overhead.

Haystack detected 540 objects within its $0.058^{\circ}$ half-power beam-width during the 1.9-hour pass for a detection rate of 290 detections per hour. Approximately one-third more detections exceeded the Haystack signal-to-noise threshold, but these were deemed to have passed through a side-lobe or through the main beam and outside the half power beam-width. Prior to the Fengyun break-up, the background detection rate for Haystack at these elevation angles was 5 to 10 detections per hour. Figure 5 shows the size distribution for the Haystack data collected during the orbit plane tracking pass.

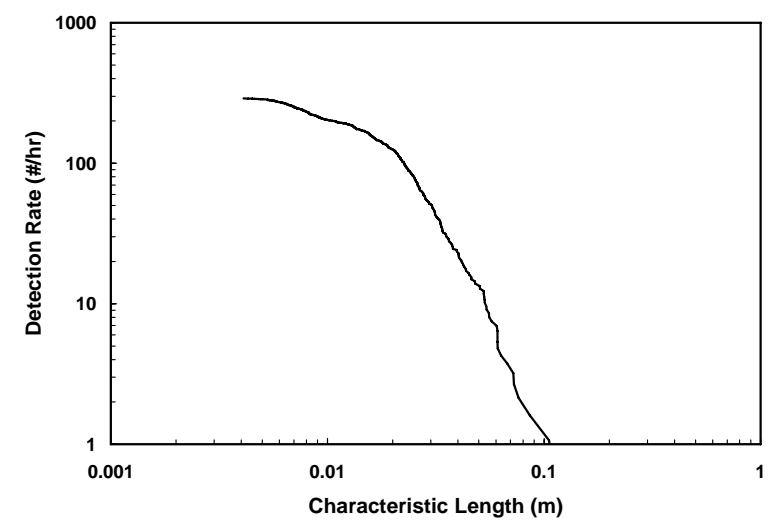

Fig. 5. Cumulative size distribution of Fengyun-1C debris detected during 1.9 hours of orbit plane tracking by the Haystack radar on 12 January 2007. 
Figure 6 depicts the time and altitude of each detection. This plot gives an indication of how the debris is distributed along the orbit plane. Unfortunately, the densest part of the cloud is near the start and end of the pass where the orbit plane is farthest from the radar and, hence, the sensitivity of the radar is at its lowest.

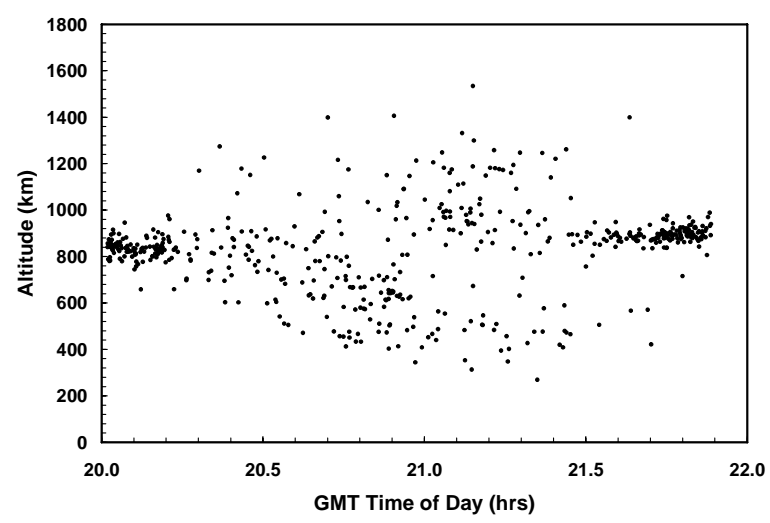

Fig. 6. Altitude and time for each Fengyun-1C debris detected during 1.9 hours of orbit plane tracking by the Haystack radar on 12 January 2007. This figure gives an indication of how the debris were distributed along the orbit plane 24 hours after the break-up.

In the weeks and months following the break-up event, the Fengyun debris were detected by Haystack during its normal staring operations for statistically sampling the debris population. In this operational mode, the radar is pointed at an elevation of $75^{\circ}$ and an azimuth of $90^{\circ}$, and objects passing through the beam between altitudes of 350 to $1800 \mathrm{~km}$ are detected. Figure 7 shows a plot of inclination vs. time-of-day for 184 hours of data collected between January and May, 2007. Since the Fengyun-1C spacecraft was in a sun-synchronous orbit, its orbit plane passed through the Haystack beam at the same time each day ( 2040 GMT). A second pass occurred at $\sim 0950$ GMT, but Haystack was not operated for debris detections during that time window. As noted previously, Haystack detected much smaller objects than did the SSN.

The inclination spread evident in Figure 7 and associated with the Fengyun-1C plane crossing is larger than that seen in the cataloged debris, as expected with the inclusion of smaller debris. Inclination in the Haystack data is estimated by

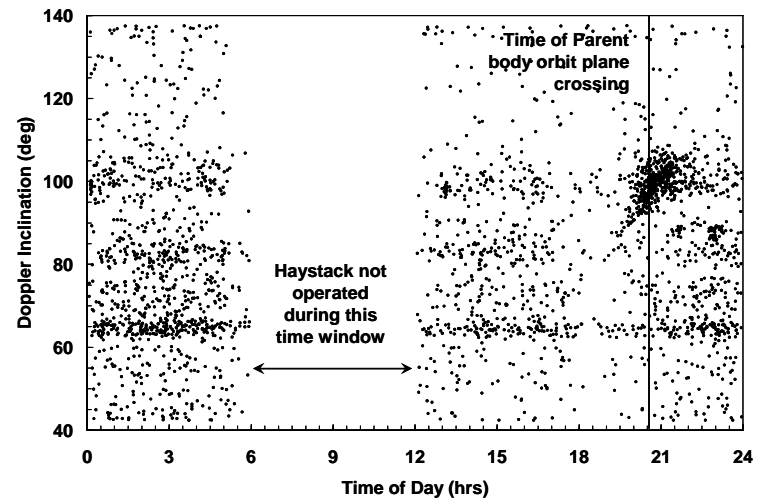

Fig. 7. Haystack staring data collected from January to May, 2007. Many of the detections near $100^{\circ}$ inclination and 2100 hrs are Fengyun-1C debris.

looking at the range rate of an object and assuming a circular orbit. Although this assumption introduces some uncertainty, it does not account for the entire inclination spread. The Haystack data also shows an asymmetry in inclination. Approximately two-thirds of the detections associated with Fengyun-1C (based on detection time and inclination) show inclinations higher than the pre-impact parent, i.e., higher than 98.65 degrees.

The detection rate, or number of detections per hour, was calculated for each half hour in the diurnal day and is shown in Figure 8. The background detection rate away from the time of

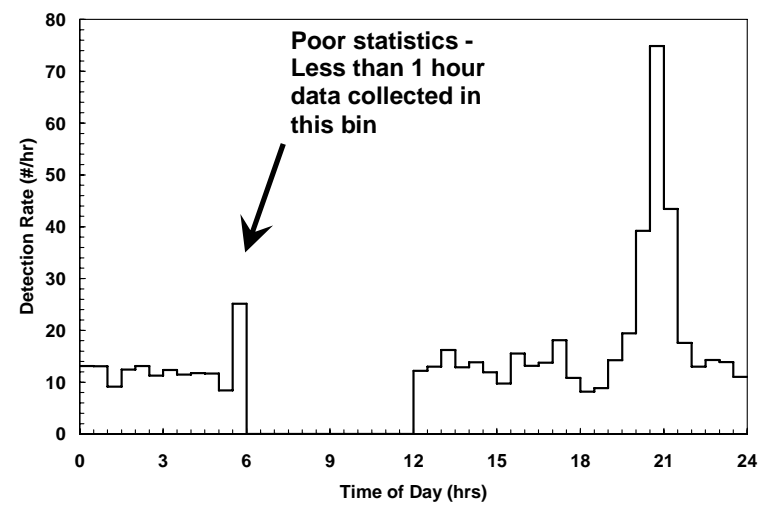

Fig. 8. Diurnal detection rate for the Haystack $75^{\circ}$ East staring data shown in Figure 7. The peak detection rate corresponding to passage of the Fengyun-1C cloud through the radar beam is 6 times higher than the background rate of 12.4 detections per hour. 
the Fengyun-1C orbit plane passage was 12.4 detections per hour, whereas the peak detection rate for the Fengyun-1C debris was 75 detections per hour for the half hour containing the plane passage.

The Haystack staring data and the orbit plane tracking data will be used to estimate the total number of fragments larger than $1 \mathrm{~cm}$ in characteristic length along with the size and velocity distributions associated with the Fengyun-1C breakup. Later, data from the more sensitive Goldstone radar could provide information on debris as small as $2-3 \mathrm{~mm}$.

\section{CHANGES IN THE NEAR-EARTH ENVIRONMENT}

The deposition of more than 2000 Fengyun-1C debris in low Earth orbit (LEO) represented an immediate increase of more than one-third in the cataloged LEO debris environment, a population which had taken 50 years to accumulate. The

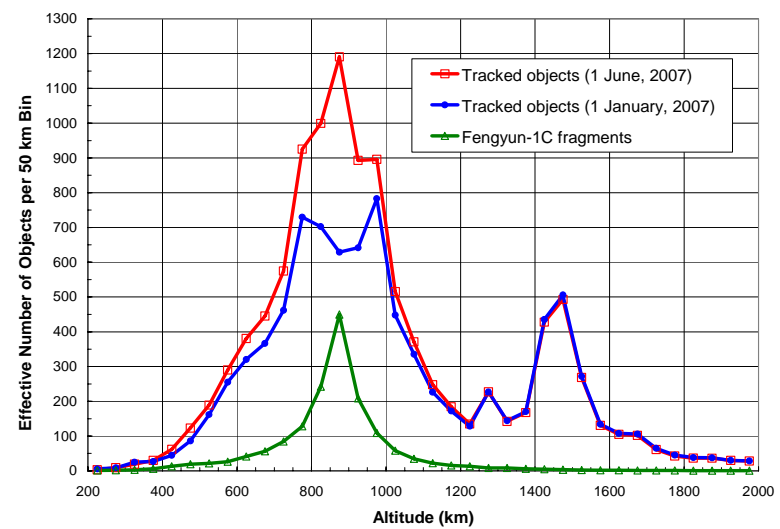

Fig. 9. Distribution of Fengyun-1C debris and effect on the LEO population.

majority of the new debris were found near the altitude of the Fengyun-1C break-up. Figure 9 compares the relative LEO object populations, as determined by the SSN, before and after the break-up. The "effective" number of objects reflects the time that each debris spends in a specific altitude range, i.e., $50 \mathrm{~km}$ altitude bin.
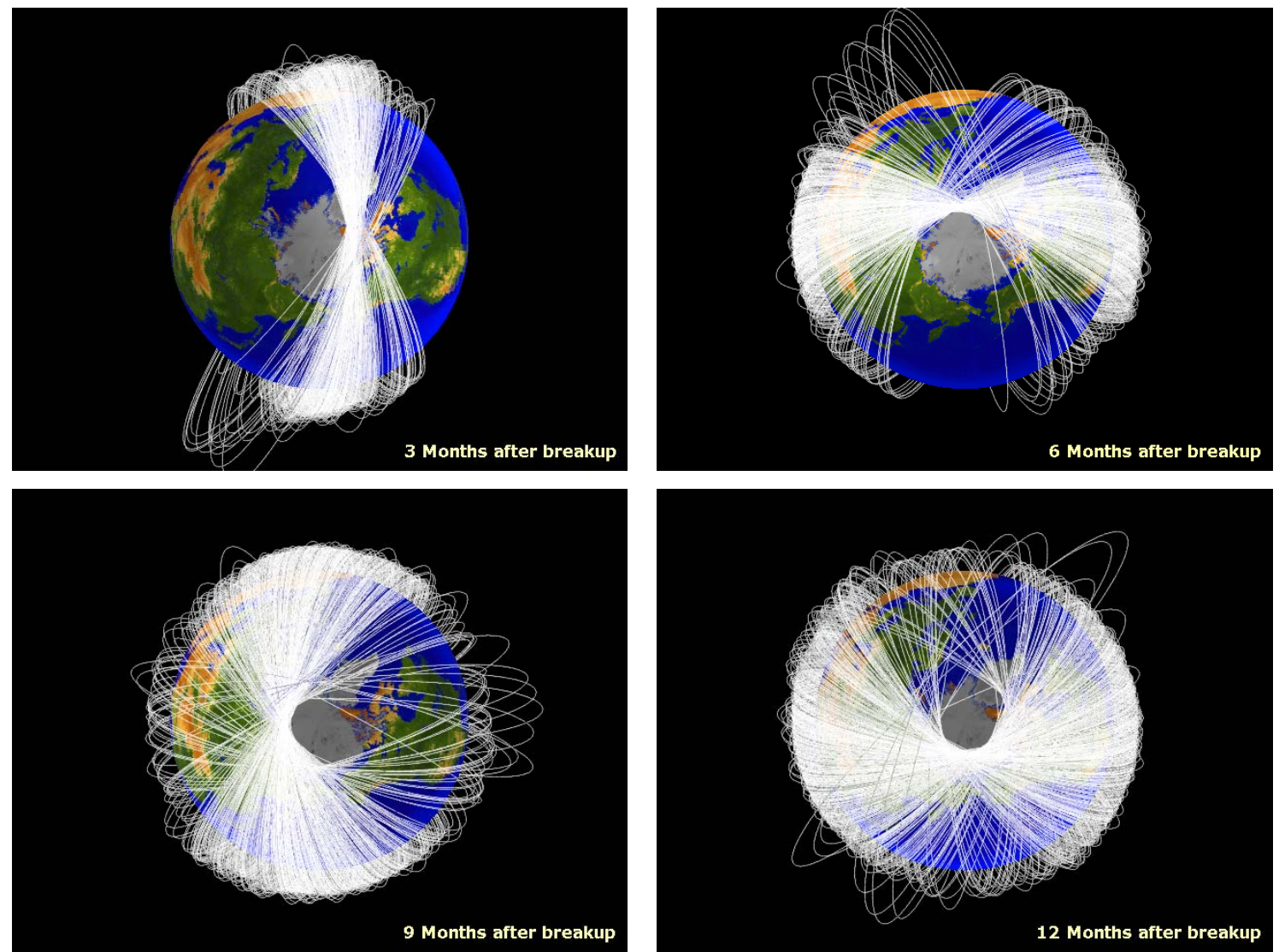

Fig. 10. Evolution of the Fengyun-1C debris orbit planes. 
In addition to a distribution of debris across altitudes, the orbital planes of the debris slowly spread due to the variation in orbital periods. Immediately after the break-up, the debris were constrained within a very narrow range of inclinations and right ascensions, appearing to reside in a thin disk. However, differential rates of precession lead over time to a much broader dispersion of the debris in LEO. Figure 10 illustrates the Fengyun-1C debris planes at 3month intervals after the break-up.

By 11 July 2007, six months after the break-up of Fengyun-1C, only 13 cataloged debris had fallen back to Earth. The majority of the debris will remain in orbit for decades, some even lasting more than one hundred years.

Figure 11 is a projection of the decrease in the number of large debris $(>10 \mathrm{~cm})$ in orbit over time, based upon preliminary debris $\mathrm{A} / \mathrm{M}$ assessments and typical future solar activity variations. The greater number of observed debris off-sets this apparent faster than normal decay rate, leaving the overall probability of collision estimates virtually unchanged from model predictions. Debris smaller than $10 \mathrm{~cm}$ are expected to reenter the atmosphere slightly more rapidly, but their substantially greater numbers will result in even greater collision risks to operational spacecraft in LEO.

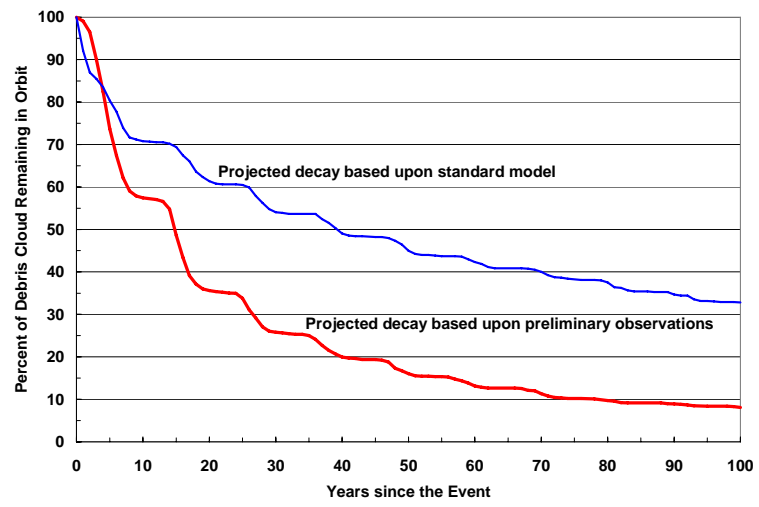

Fig. 11. Projected gradual orbital decay and reentry of tracked debris from Fengyun-1C. The apparent accelerated rate of the actual debris is off-set by the greater number of debris, in comparison with model prediction values.

\section{NEAR- AND LONG-TERM COLLISION EFFECTS}

Due to the large number of tracked Fengyun-1C debris, close approaches between the debris and operational spacecraft throughout LEO are common. In fact, some collision avoidance maneuvers had already been executed within the first six months after the test. For example, NASA's Terra spacecraft, in a nearly circular orbit at 705-km mean altitude, was commanded on 22 June to avoid a very close approach (19 meters) from a 35-cm fragment from Fengyun1C. Just a few days later, the International Space Station was prepared to make a similar maneuver to avoid another piece of Fengyun-1C debris, but the maneuver was canceled shortly before the planned engine ignition when the miss distance was determined to be acceptable.

The long-term impact of Fengyun-1C debris to the LEO environment can be assessed with the NASA orbital debris evolutionary model LEGEND (a LEO-to-GEO Environment Debris model). The model can mimic the historical debris environment based on the historical launch record and breakup events and has the capability to project the environment into the future based on reasonably assumed launches and an algorithm to predict collision activities in the environment ${ }^{5}$. Two sets of LEGEND simulations have been carried out. Both included a historical component between 1957 and the end of 2006 and a future projection through 2106. The only difference between the two simulations was the Fengyun-1C breakup was added to the end of the historical period for one of them. A non-mitigation ("business-as-usual") scenario was assumed during the projection period, and the 1999-to-2006 launch cycle was repeated. Each simulation included 100 Monte Carlo runs to ensure good statistical sampling of the future environment.

The $10 \mathrm{~cm}$ and larger LEO populations from the two simulations are shown in Figure 12. There is a noticeable difference between the two. As mentioned in Section 2.1, the number of large (10 $\mathrm{cm}$ and larger) fragments generated by the 
Fengyun-1C breakup far exceeded that predicted by the NASA standard breakup model for a nominal event. Since the LEGEND simulation (bold curve) was based on fragments generated by the NASA breakup model, the actual impact of the Fengyun-1C breakup to the environment should be worse than what is shown in the figure. When more observational data are available to better characterize the Fengyun-1C fragments, an updated assessment will be performed to more accurately assess the damage of the event to the LEO environment.

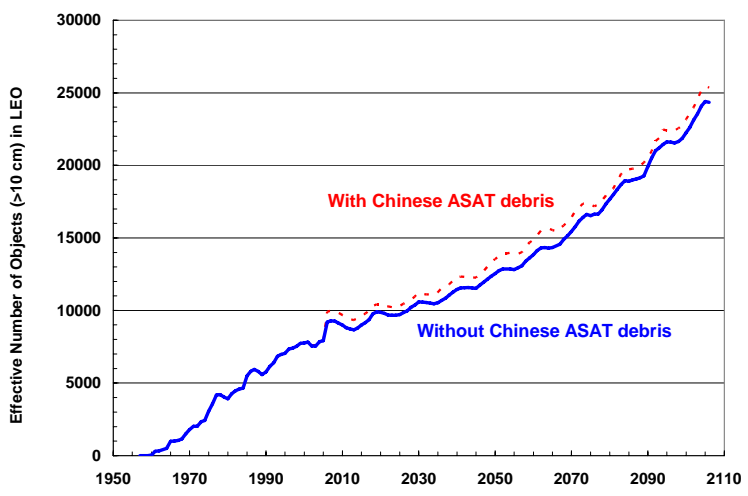

Fig. 12. The LEO populations (objects $10 \mathrm{~cm}$ and larger) from the two LEGEND simulations. The difference between the two curves is due to the Fengyun-1C break-up. Note the fragments were generated based upon the NASA standard breakup model. Data from the SSN indicate that the number of fragments actually generated from the event might exceed the nominal debris population predicted by the model.

\section{CONCLUSIONS AND SUMMARY}

This paper has presented clear evidence of the magnitude of the break-up of the Fengyun-1C spacecraft and its substantial impact on the nearEarth environment. In terms of cataloged debris, this event has constituted the worst single fragmentation in the history of the space age, and its effects will be very long-lived.

Prior to this event, no in-space ASAT test had been conducted in over 20 years. From 1968 through 1982, the former Soviet Union conducted a series of 20 ASAT tests, of which nine resulted in the creation of orbital debris clouds. A total of 736 debris from those tests have been cataloged by the SSN with about $40 \%$ still remaining in orbit.

An important aspect of those tests was an apparent change in the second part of the program to minimize orbital debris. The first phase of testing resulted in six orbital debris clouds from seven tests. The second phase produced only three orbital debris clouds from 13 tests. Engagement geometries in the second phase typically resulted in the generation of debris on reentering, instead of orbital, trajectories.

The short-lived testing of an ASAT device by the U.S. in the 1980's resulted in only one direct impact in 1985 with 285 cataloged debris, of which none remain in orbit. In fact, the original test program envisioned using inflated balloons for targets to minimize the creation of orbital debris. Only when the development of the dedicated targets was delayed due to technical problems did officials select a single low altitude (525 km) resident space object as a target. Other tests were conducted using phantom targets, i.e., stars, which resulted in zero debris.

Based, in part, upon the above experience of the former Soviet Union and the U.S., the space debris mitigation guidelines of both the InterAgency Space Debris Coordination Committee (IADC) and the United Nations Committee on the Peaceful Uses of Outer Space (COPUOS) strongly recommend avoiding the creation of long-lived debris in any deliberate fragmentation in space:

\section{IADC Space Debris Mitigation Guidelines, paragraph 5.2.3: Avoidance of intentional destruction and other harmful activities}

"Intentional destruction of a space system (self destruction, intentional collision, etc.) and other harmful activities that may significantly increase collision risks to other systems should be avoided. For instance, intentional breakups should be conducted at sufficiently 
low altitudes so that orbital fragments are short live."

\section{United Nations Space Debris Mitigation Guideline 4: Avoid intentional destruction and other harmful activities.}

"Recognizing that an increased risk of collision could pose a threat to space operations, the intentional destruction of any on-orbit spacecraft and launch vehicle orbital stages or other harmful activities that generate long-lived debris should be avoided.

"When intentional break-ups are necessary, they should be conducted at a sufficiently low altitudes to limit the orbital lifetime of resulting fragments.",

The deliberate fragmentation of the Fengyun-1C spacecraft was in contradiction to the above guidelines. Following criticism by several countries concerned about the test, Chinese National Defense Minister Cao Gangchuan was reported to have stated on 12 February that no further such tests were planned. ${ }^{8}$

The nature and high altitude of the test have resulted in an intense orbital debris cloud with considerable persistence. The apparent greaterthan-expected number of large debris produced poses a real risk to operational spacecraft throughout LEO, which is only partially offset by a slightly higher anticipated rate of reentry for the debris. A future increase in the total debris environment is likely due to collisions between Fengyun debris and existing larger resident space objects.

\section{REFERENCES}

1. Covault, C., "Chinese Test Anti-Satellite Weapon", Aviation Week and Space Technology website, 17 January 2007.

2. anom., "First Confirmation by PRC of Test of Anti-Satellite Weapon", Zhongguo Tongxun She News Agency, Hong Kong, 23 January.

3. Covault, C., "China's Asat Test Will Intensify U.S.-Chinese Faceoff in Space”, Aviation Week and Space Technology, 22 January 2007, p. 24.

4. Xu, Yu-lin et. al., "A Statistical Size Estimation Model for Haystack and HAX Radar Detections", IAC-05-B6.1.02, 56 ${ }^{\text {th }}$ International Astronautical Congress, Fukuoka, Japan, October 2005.

5. Liou, J.-C., "Collision Activities in the Future Orbital Debris Environment”, Adv. Space Research, Vol. 38, No. 9, 2006, pp. 2102-2106.

6. anom., IADC Space Debris Mitigation Guidelines, IADC-02-01, 15 October 2002.

7. anom., Report of the Scientific and Technical Subcommittee on its forty-fourth session, held in Vienna from 12 to 23 February 2007, A/AC.105/890, Appendix IV, United Nations, 6 March 2007.

8. anom., Kyodo News Service, Tokyo, 12 February 2007. 